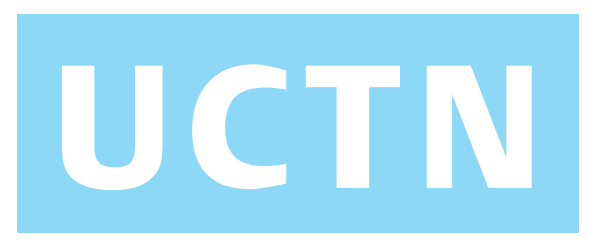

\title{
Glutaraldehyde colitis
}

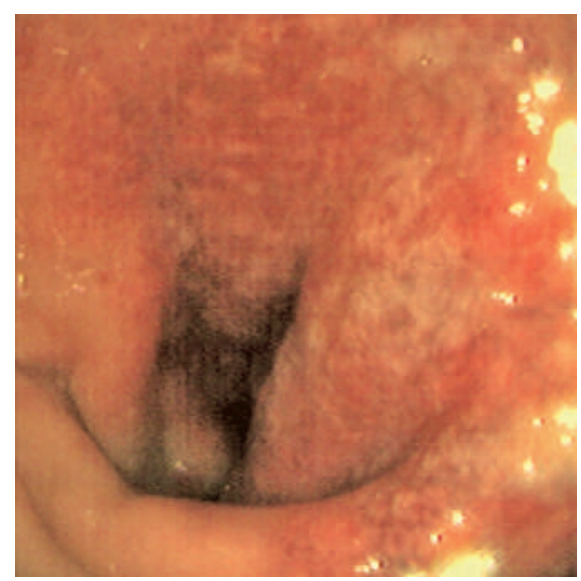

Figure 1 At sigmoidoscopy the patient was found to have markedly inflamed mucosa without any ulceration.

A previously healthy, 69-year-old woman underwent a screening colonoscopy in November 2005. A 1-cm polyp was found in the sigmoid colon and was resected by snare polypectomy. The patient developed mild lower abdominal pain and passed some bloody stools 6 hours after the procedure. She was subsequently admitted and a sigmoidscopy was performed without bowel preparation in order to evaluate the postpolypectomy bleeding. The sigmoidscopy revealed markedly inflamed mucosa, but no ulceration (Figure $\mathbf{1}$ ). During the same period, a total of six patients developed similar symptoms, symptoms that have not been encountered in our endoscopy unit in the past 20 years. Abdominal computed tomography performed in another similar patient (aged 72 years) showed involvement of the rectum and sigmoid colon only (Figure 2). All the microbiological studies in these patients were negative. The patient's symptoms resolved 3 days after bowel rest and intravenous fluid supplementation.

It was discovered that a defect in one of the endoscope-cleansing machines was causing retention of $2 \%$ glutaraldehyde disinfectant in the endoscope channels. No similar case has occurred in the 6 months since this defect was rectified. A diagnosis of glutaraldehyde colitis was made.

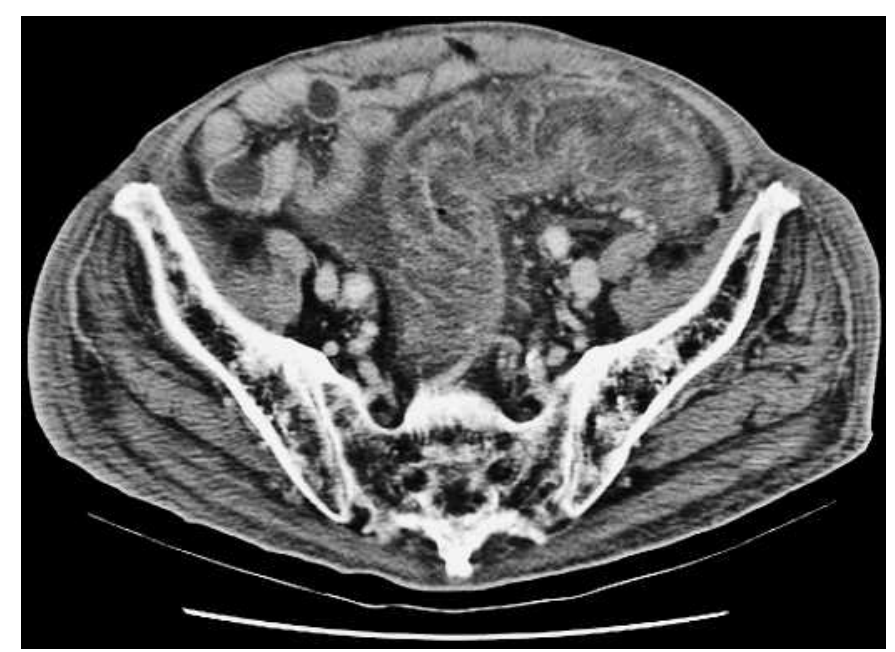

Figure 2 Abdominal computed tomography in another affected patient revealed marked mural thickening in the rectum and sigmoid colon.

Glutaraldehyde, a disinfectant commonly used in flexible endoscopes, is a rare cause of postcolonoscopic colitis. In 1986, Castelli et al. [1] reported the first outbreak of postcolonoscopy colitis, and this was attributed to retained glutaraldehyde in the colonoscope channel. Patients typically develop abdominal pain, tenesmus, and bloody diarrhea within 48 hours of the endoscopic examination [1-4]. Repeat endoscopy may reveal edema, hyperemia, friability, or pseudomembrane formation in the involved colon. Biopsy can reveal nonspecific inflammation or findings that mimic ischemic colitis. Computed tomographic findings are also nonspecific, and can include mural thickening and sometimes pericolonic inflammatory stranding $[5,6]$. Most of these findings are confined to the distal colon. The symptoms usually subside spontaneously after bowel rest.

Endoscopists should be aware of this iatrogenic complication in patients presenting with acute colitis, especially in those with a known history of recent endoscopic examination. Once such a complication occurs, identification of the cause is mandatory in order to avoid further cases of chemical colitis [1-4]. Possible causes of this complication include failure to follow the rinsing protocol, or defects in the endoscope, cleansing machine, or connecting tube.
Endoscopy_UCTN_Code_CCL_1AD_2AD Endoscopy_UCTN_Code_CPL_1AN_2AG

\section{H.-H. Yen, Y.-Y. Chen}

Department of Gastroenterology, Changhua Christian Medical Center, Changhua, Taiwan.

\section{References}

${ }^{1}$ Castelli M, Qizilbash A, Seaton T. Post-colonoscopic procitis [Abstract]. Am J Gastroenterol 1986; 81: 887

2 West AB, Kuan SF, Bennick M, Lagarde S. Glutaraldehyde colitis following endoscopy: clinical and pathological features and investigation of an outbreak. Gastroenterology 1995; 108: 1250-1255

${ }^{3}$ Rozen P, Somjen GJ, Baratz M et al. Endoscope-induced colitis: description, probable cause by glutaraldehyde, and prevention. Gastrointest Endosc 1994; 40: 547-553

${ }^{4}$ Caprilli R, Viscido A, Frieri G, Latella G. Acute colitis following colonoscopy. Endoscopy 1998; 30: $428-431$

${ }^{5}$ Birnbaum BA, Gordon RB, Jacobs JE. Glutaraldehyde colitis: radiologic findings. Radiology 1995; 195: 131 - 134

${ }^{6}$ Zissin R, Gayer G, Maor-Kendler Y. CT findings of glutaraldehyde colitis: a report of two cases. Clin Radiol 1999; 54: $123-125$

\section{Corresponding author}

\section{Y.-Y. Chen, M.D.}

Changhua Christian Medical Center

135 Nanhsiao Street Changhua

Changhua 500 - Taiwan

Fax: $\quad+886-4-7228289$

E-mail: 27716@cch.org.tw

DOI: $10.1055 / \mathrm{s}-2007-966087$ 